\title{
Routing Protocol Using (m,k)-firm Scheduling Scheme to Ease Hole Problems in WSNs
}

\author{
Jaehyun Nam \\ Silla University, Department of Information Technology, \\ Busan, Korea \\ jhnam@silla.ac.kr
}

\begin{abstract}
Wireless Sensor networks require a routing protocol that could efficiently use inherent limited energy resource of sensor nodes. All sensor nodes first sense data in many-to-one sensor network, and transmit it directly to the sink through multi-hop transmission. Since this method transmits data through a specific path within network, the nodes in the corresponding path use energy faster than other nodes and the hole problems are generated within the network. Therefore, we suggests a routing protocol to ease routing hole problems that could occur due to intensive energy consumption of a specific path during the transmission of sensed data from a sensor node to neighbor nodes. The proposed routing protocol enhanced energy efficiency of all nodes in a network by inducing data transmission from the source to a sink through different pathways. In the proposed protocol, a next hop was decided based on $(m, k)$-firm scheduling technique, energy expenditure in each node, and transmission delay among nodes. Performance evaluation through simulations verified improved performance of the proposed protocol compare to conventional routing protocols.
\end{abstract}

Keywords: energy hole problems, uneven energy consumption, $(m, k)$-firm scheduling

\section{Introduction}

Thousands or millions of small and inexpensive low power sensors are used in monitoring a huge field. Each sensor possesses limited sensing region, processing power, and energy. These sensors detect information on a certain environment and transmit the detected data through wireless channels. Sensors consume energy in processing and transmitting sensed events [1]. However, efficient energy use is crucial for sensor nodes operated by batteries to extend the overall network lifespan. A large number of studies to use efficient energy have been performed on different layers of protocols. In MAC layer protocols, CSMA and TDMA access schemes and a hybrid method of CSMA and TDMA have been investigated [2-4]. For efficient energy use at network layer level, routing protocols that recognize energy have been proposed [5-6]. Although those protocols can save energy to some degree through protocols that use sleep schedules [7], energy efficiency decreases in these protocols when traffic load is unevenly distributed over the nodes in a network. Thus, no studies investigated how traffic load is distributed in a multi-hop WSN and the effect of traffic load on network lifespan.

Nodes in a sensor network conduct sensing and communicating tasks simultaneously. To perform these tasks, nodes must be capable of performing the functions of sensing some phenomena and communicating with neighboring nodes for the transmission of sensed data to the sink. However, a wide range of abnormal situations could occur damaging the functions of wireless sensor network in the actual field. These problems incur coverage holes, routing holes, jamming holes, and sink/back/worm holes problems [8-12]. Holes occur because of 
empty spaces created while deploying sensor nodes or sensor nodes destroyed by environmental causes. Nodes along the border area of hole are most likely to be used as routers compare to other ordinary nodes in a random communication session. For this reason, nodes deployed around the border of holes are characterized by rapid depletion of energy compare to other standard nodes [13]. Therefore, load balancing is considerably crucial in sensor networks with high-density nodes and inadequate communication bandwidth. The load balancing prevents rapid consumption of energy spent by specific nodes among all nodes in a network.

This paper proposes a new routing protocol to ease the problems of routing holes that occur due to intensive energy consumption in specific path while transmitting sensed data from the sensor node to neighboring nodes. Taking advantage of the (m,k)-firm model [16], it is available for our scheme to avoid the fast drain of sensor nodes with high energy consumption. To achieve this, our scheme balances the energy consumption by sending the traffic generated each node through multipath routing instead of sending through the same path. It improves energy efficiency of all nodes in a network by inducing data transmission from the source to a sink through different path. The simulations demonstrated that each node in the proposed protocol consumed less energy compare to that of existing routing protocols by efficiently distributing traffic load more evenly in the network.

The paper is organized as follows. Section 2 contains related works. Section 3 introduces the hole problem and the concept of ( $\mathrm{m}, \mathrm{k})$-firm scheduling scheme, and presents our proposed routing protocol. Sections 4 discuss performance evaluation of the proposed routing protocol against existing routing protocols in simulation. Section 5 concludes the paper.

\section{Related Works}

In this section we describe various proposed solutions for finding and fixing holes in sensor networks.

Wang et al. in [8] is to maximize the coverage of a given target area with constraints on deployment time, the distance the sensors have to travel to maximize coverage and the complexity of the protocol. Simulation results presented in [8] shows that the Minimax algorithm outperform the other two proposed algorithm in achieving maximum coverage with little increase in computational overhead. However, on average, Minimax moves the sensors longer distance than the other two algorithms to achieve this higher level of coverage.

Howard et al. [9] proposed a potential fields based approach for self-deployment of mobile sensor networks. Nodes are treated as virtual particles and the virtual forces due to potential fields repel the nodes from each other and obstacles. However, extensive simulation/experimental studies have not been conducted to test the sensitivity of the approach to changes in communication and sensing ranges and different network sizes etc.

Intanagonwiwat et al. [10] were the first to propose a data centric communication protocol for wireless sensor networks called Directed Diffusion (DD). DD can be tuned to work as a multipath protocol if alternate paths are kept alive by sending reinforcement with different interval attributes to different neighbors.

The interest flooding overhead in DD becomes high when the number of sinks increases [11]. Also the event flooding proposed in push diffusion is only viable when number of sinks interested in the event is much more than the number of sources. Braginsky et al. in [12] proposed Rumor Routing, to address these overheads.

Reactive routing algorithms such as AODV and DSR maintain routing information for small subset of possible destination. If no route is available for new destination, a route discovery process is invoked $[14,15]$. A node uses flooding to discover new paths. In sensor 
networks where thousands of nodes communicate with each other, broadcast storms may result in significant power consumption and possibly a network meltdown.

\section{Routing Protocol to Ease Routing Hole Problem}

\subsection{The Hole Problem}

A routing hole consists of a region with nodes that cannot participate in the actual routing of data or no usable nodes for data transmission due to various reasons in sensor networks. It could be created due to spaces formed while deploying sensor nodes, manufacturing problem in nodes, battery depletion, and destruction of sensor node caused by external factors.

Routing holes show the local minimum phenomenon [13]. This problem is resulted from the absence of neighboring nodes located near the destination. In case of the local minimum phenomenon, a data packet is transmitted along the border of hole area. If multiple communication sessions had to bypass holes concurrently, the probability of collision among nodes increases because nodes around the border of holes are more likely to be shared by several communication sessions. Furthermore, the holes are very likely to be expanded because energy is used up faster in nodes located around the border of holes compare to other nodes within a network.

\subsection{Distance Based Priority (DBP)}

DBP was a dynamic priority assignment mechanism for jobs under $(\mathrm{m}, \mathrm{k})$-firm constraint [16]. The basic idea of DBP algorithm is as follows: the closer the stream to a failure state, the higher its priority is. A failure state occurs when the stream's $(\mathrm{m}, \mathrm{k})$-firm requirement is violated. That is, the deadlines of at least $\mathrm{m}$ out of any consecutive $\mathrm{k}$ packets must be met For each stream source, which requires an $(\mathrm{m}, \mathrm{k})$-firm, the priority is assigned based on the number of consecutive deadline misses that leads the stream to violate its (m,k)-firm requirement. This number of deadline misses is referred to as distance to failure state from current state.

The k-sequence is a word of $\mathrm{k}$ bits ordered from the most recent to the oldest job in which each bit keeps memory of whether the deadline is missed (bit $=0)$ or met (bit=1). Each new job causes a leftward shift of all the bits, the leftmost exists from the word and is no longer considered, while the rightmost will be a 1 if the job has met its deadline or a 0 otherwise.

The priority of its job at a given instant can be assigned with the distance of the current ksequence to a failure state. Evolving the k-sequence can determine the violating distance of the job. That is left shift the k-sequence and adding in the right side 0s until the evolved ksequence violates $(\mathrm{m}, \mathrm{k})$-firm of the job, and the number of added $0 \mathrm{~s}$ is the priority. If a job stream is already in failure state, the highest priority 0 is assigned.

Normally, for a job $\tau$ with constraint $\beta=(\mathrm{m}, \mathrm{k})$-firm, let priority $\operatorname{VD}^{\beta}(\tau)$ denote its violating distance, we get

$$
V D^{\beta}(\tau)=k-l(m, s)+1
$$

where s denote the state of the previous $k$ sequence jobs of $\tau, l(m, s)$ denote the position (from the right) of the nth meet (or 1) in the s. For example, considering a stream with $(4,6)$-firm constraint, the current job is set the priority of 3 if its previous six consecutive jobs construct the state of (101111). 


\subsection{Modified Load Balancing Routing Protocol (MLBRP)}

In our prior work [17], a load balancing routing protocol (LBRP) was presented to balance the energy consumption in each sensor node using a scheduling policy called distanced-based priority (DBP). In this paper, we propose MLBRP to advance our prior work. In LBRP, only $(\mathrm{m}, \mathrm{k})$-firm violating distance and end-to-end delay were taken into account when selecting adjacent nodes for sensed data transmission. In contrast, MLBRP considers energy spent for data transmission together, in addition to $(\mathrm{m}, \mathrm{k})$-firm violating distance and delay to select next hop. Although the energy consumption among nodes is not taken into consideration in the LBPR technique, this is considered in the MLBRP because energy expenditures among nodes are associated with the distance from the source to a sink.

In the MLBRP, every node broadcasts a beacon to neighboring nodes regularly like other geographic routing algorithms. Beacon is used for exchanging location information between neighboring nodes. Moreover, a sink node broadcasts Back Advertisement Message (BAM) to other sensor nodes to form transmission pathways between the source and a sink. In this process, minimum energy overhead required for data transmission between nodes is transferred in BAM. This information is stored in cost field within a flow table. The fields of BAM consist of a sink ID and an energy cost. Moreover, every node maintains a flow table to store information obtained from beacons.

Each entry in the table has the following fields:(Neighbor ID, Cost, Delay, $V D^{\beta}$ ). The cost field of the flow table at each node is the minimum energy overhead required in forwarding a data packet from a corresponding node to a sink node. In this paper, we suppose that the cost of sending data from each node to nearby neighbors could be estimated. Cost values are small for nodes adjacent to the sink node and large for nodes apart from the sink node. Delay is calculated by dividing the advance in distance from the next hop node $i$ by the estimated delay to forward a packet to node $i$.

At the arrival of the data packets, the path with the smallest metric value is selected first from the flow table. Equation (2) shows the calculation of metric value.

$$
\text { metric }=a \cdot V D^{\beta}+b \cdot \operatorname{Cost}+c \cdot \text { Delay }
$$

The first term in the equation (2) represents the (m,k)-firm violating distance, the second term denotes energy used for data transmission to the next hop, and the last term means delay. The study applied $a=0.5, b=0.3$, and $c=0.2$. This was because $(\mathrm{m}, \mathrm{k})$-firm violating distance was considered significant in order to distribute paths for data transmission in metric calculation.

If a node fails to transmit a packet to one neighbor due to a collision, a path having a second smallest metric value is selected. Thus, the path with the second smallest metric value is decided as the next hop when collisions or $m$ consecutive transmissions occur.

\section{Evaluation}

In this section, we evaluated the performance of the proposed protocol using ns-2 simulator from the aspects of maximum energy consumption of nodes. In simulation, we compared the performance of the proposed routing protocol and DSDV routing protocol. The structure of nodes is composed as shown in Figure 4, and simulation parameters are presented in Table 1. Network topology used in the simulation environment was uniform topology deployed with 100 nodes in an area of $200 \mathrm{~m}$ X $200 \mathrm{~m}$.

Figure 1 is the comparison of energy expenditures per unit hour when MLBRP, LBRP, and DSDV algorithms were operated in the simulation environment. As shown in the Figure, the amount of energy consumption in the MLBRP technique decreased as the data traffic of sensor nodes increased. In the DSDV algorithm, data packets were continuously transmitted 
to the decided path once next hop was determined only by considering the best path. This method eventually generates routing holes by unevenly using energy in specific nodes. However, MLBRP alleviates the energy depletion in specific nodes because of considering $(\mathrm{m}, \mathrm{k})$-firm violating distance, delay, and energy spent for data transmission to select next hop.

\section{Table 1. Simulation settings}

\begin{tabular}{c|c}
\hline Routing & DSDV, LBRP \\
\hline Mac Layer & 802.11 \\
\hline Propagation model & TWO-RAY \\
\hline Bandwidth & $200 \mathrm{~Kb} / \mathrm{s}$ \\
\hline Transmission power & $24 \mathrm{~mW}$ \\
\hline Receive power & $10 \mathrm{~mW}$ \\
\hline Idle power & $10 \mathrm{~mW}$ \\
\hline
\end{tabular}

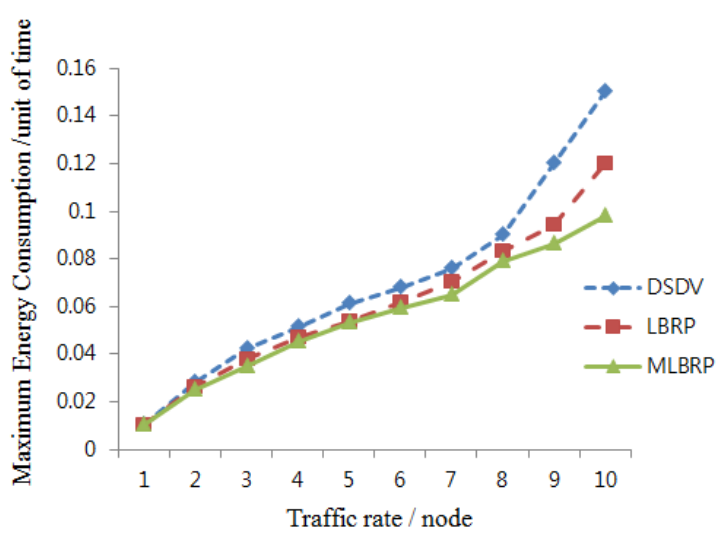

Figure 1. Comparison of the energy consumption between LBRP and DSDV

\section{Conclusion}

Sensor networks are a fundamental technology enabling human-computer interaction by exchanging data over wireless channel among numerous tiny sensor nodes that are deployed all over the monitoring places. Expansion of network lifespan has emerged as the most critical issue by reducing energy consumption of sensor nodes in sensor networks. For this reason, a large number of studies on efficient energy use have been performed at MAC layer and network layers. We proposes a routing protocol that enables even energy consumption of nodes during data transmission from the source to a sink in a sensor network composed of fixed sensors. To avoid the fast drain of sensor nodes with high energy consumption, we was to take advantage of the $(\mathrm{m}, \mathrm{k})$-firm model. The proposed protocol prevents energy depletion of specific nodes by sending the traffic generated each node through multipath routing instead of sending through the same path. Performance evaluation with simulation showed that the average energy consumption in the proposed protocol was lower compare to existing routing protocols. 


\section{References}

[1] I. F. Akyildiz, W. S.Y. Sankarasubramaniam and E. Cayirci, "A survey on sensor networks", IEEE, Communications Magazine, (2002), pp. 102-114.

[2] M. Miller and N. Vaidya, "A mac protocol to reduce sensor network energy consumption using a wake-up radio", IEEE, Transactions on Mobile Computing, vol. 4, no.3, (2005), pp. 228-242.

[3] W. Ye, J. Heidemann and D. Estrin, "Medium access control with coordinated adaptive sleeping for wireless sensor networks", IEEE/ACM, Transactions on Networking, vol. 12, no. 3, (2004), pp.493-506.

[4] T. V. Dam and K. Langendoen, "An adaptive energy-efficient MAC protocol for wireless sensor networks", ACM SenSys 2003, Los Angeles, CA, (2003) November, pp.171-180.

[5] R. C. Shah and H. M. Rabaey, "Energy aware routing for low energy ad hoc sensor networks", IEEE/WCNC, Orlando, FL, USA, (2002) March.

[6] J. Chang and L. Tassiulas, "Maximum lifetime routing in wireless sensor networks", IEEE/ACM, Transactions on Networking, vol. 12, no. 4, (2004) August, pp. 609-619.

[7] C. F. Chiasserini and M. Garetto, "An Analytical Model for Wireless Sensor Networks with Sleeping Nodes", IEEE, Transactions on Mobile Computing, vol. 5, no. 12, (2006) December, pp.1706-1718.

[8] G. Wang, G. Cao and T. La Porta, "Movement-assisted sensor deployment. In IEEE INFOCOM 2004", (2004) June.

[9] A. Howard, M. J. Mataric and G. S. Sukhatme, "Mobile sensor network deployment using potential fields: A distributed, scalable solution to the area coverage problem", In 6th International Symposium on Distributed Autonomous Robotics Systems (DARS02), (2002) June.

[10] C. Intanagonwiwat, R. Govindan and D. Estrin, "Directed diffusion: A scalable and robust communication paradigm for sensor networks", In Proceedings of the Sixth Annual ACM/IEEE MobiCom '2000, (2000) August, pp. 56-67.

[11] J. Heidemann, F. Silva and D. Estrin, "Matching data dissemination algorithms to application requirements", In Proceedings of the ACM SenSys '03, (2003).

[12] D. Braginsky and D. Estrin, "Rumor routing algorithm for sensor networks", In 1st ACM WSNA '02, (2002) September.

[13] B. Karp and H. T. Kung, "GPSR: Greedy Perimeter Stateless Routing for Wireless Sensor Networks", Proc. MobiCom, (2000).

[14] C. Perkins, E. B.-Royer, S. Das and Ad hoc, "On-Demand Distance Vector (AODV) Routing", http://www.ietf.org/internet-drafts/draftietf-manet-aodv-13.txt, (2003).

[15] D. B. Johnson, D. A. Maltz, H. Y. Chun and J. G. Jetcheva, "The Dynamic Source Routing Protocol for Mobile Ad Hoc Networks (DSR)", Internet Draft, IETF/MANET Working Group, draft-ietf-manet-dsr-07.txt, (2002).

[16] M. Hamdaoui and P. Ramanathan, "A Dynamic Priority Assignment Technique for Streams with (m,k)-Firm Deadlines", IEEE, Transactions on Computers, vol. 44, no. 12, (1995), pp. 1443-1451.

[17] J. H. Nam, "Load Balancing Routing Protocol for considering Energy Efficiency in Wireless Sensor Network", International Workshop Database, Networking and Communication 2013, Jeju, Korea, (2013) December, pp. 28-31.

\section{Authors}

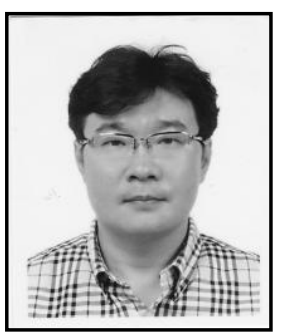

\section{Jaehyun Nam}

He received .the B.S. degree, M.S. degree and Ph.D. degree from Department of Computer Engineering, Pusan national University, Republic of Korea in 1989, 1992 and 2002 respectively. Since November 2002, he has been as associate professor at Silla University, Republic of Korea. His research interests are in ubiquitous sensor networks. 